\title{
Off-axis chromatic scanning confocal electron microscopy for inelastic imaging with atomic resolution
}

\author{
Changlin Zheng ${ }^{1}$, Ye Zhu ${ }^{1}$, Sorin Lazar ${ }^{2}$, and Joanne Etheridge ${ }^{1,3}$ \\ 1. Monash Centre for Electron Microscopy, Monash University, Victoria 3800, Australia \\ 2. FEI Electron Optics, 5600 KA Eindhoven, The Netherlands \\ 3. Department of Materials Engineering, Monash University, Victoria 3800, Australia
}

The development of electron-optical techniques for geometric and chromatic aberration correction have significantly improved the spatial resolution of transmission electron microscopy (TEM) and enhanced its capability for chemical mapping [1]. In the present work, we develop an off-axis confocal mode in a scanning transmission electron microscope (STEM) which uses the intrinsic chromatic aberration of the imaging lens system to enable fast imaging with inelastically scattered electrons without using a spectrometer [2]. Furthermore, the off-axis confocal configuration opens the possibility of obtaining depth-sensitive inelastic images for 3D imaging [3, 4].

The principle of off-axis scanning confocal electron microscopy (SCEM) is shown schematically in figure 1a. It utilizes a double-aberration corrected S/TEM to enable a large confocal angle to be employed. In the off-axis confocal mode, the electron beam is incident on the sample at an angle, $\varphi$, to the optic axis. The tilted beam introduces a lateral shift of the scattered electron wave function (elastic and inelastic) at the back focal plane of the imaging lens. Furthermore, the scattered electrons are chromatically dispersed both parallel and perpendicular to the optic axis, effectively separating electrons with different energies. In this mode, electrons with a chosen energy loss can be focused at the confocal point using the image lens (fig 1b) and detected selectively using an integrating detector to form an inelastic STEM image (fig 2c).

The method is illustrated experimentally in figure 2. A spherical-aberration corrected [5] $\operatorname{Titan}^{3}$ 80-300 S/TEM fitted with a standard Schottky field emission gun is used to examine $\mathrm{DyScO}_{3}$. First, the effectiveness of the energy filtering in this mode is tested by placing the chromatic confocal point at the entrance aperture of a post-column spectrometer. Two separate spectra corresponding to two different imaging lens focal conditions are shown overlaid in Fig 2a. In one condition, energy-loss electrons corresponding to the Scandium $\mathrm{L}_{2,3}$ edge $(\sim 400-410 \mathrm{eV})$ are focused at the chromatic confocal point, in the other the $\mathrm{Sc} \mathrm{L}_{2,3}$ pre-edge is in focus. The results prove only certain inelastically scattered electrons with selected energy loss (equal to the confocal energy) are focused at the confocal point (fig 2a). By collecting the chromatic confocal signal with a finite size BF detector, SCEM images are recorded (fig $2 b$ and $c$ ). The image formed with the Scandium $\mathrm{L}_{2,3}$ on-edge signal clearly shows atomically resolved contrast (fig 2c) while the pre-edge signal only shows faint contrast (fig 2b). The SCEM mapping is acquired with a pixel dwell time of $0.38 \mathrm{~ms}$. This is much faster than conventional EELS spectrum imaging which is limited by the read out time of the CCD. More importantly, this off-axis chromatic confocal configuration offers the potential for fast three-dimensional chemical mapping when coupled with the improved depth and lateral resolution of the incoherent confocal mode $[4,5]$.

\section{References:}


[1] K. W. Urban, J. Mayer, J. R. Jinschek, M. J. Neish, N. R. Lugg, and L. J. Allen, Phys. Rev. Lett. 110, (2013) p. 185507.

[2] C.L. Zheng, Y. Zhu, S. Lazar, J. Etheridge, Phys. Rev. Lett. 112 (2014) p. 166101.

[3] T. Wilson and C. Sheppard, Theory and practice of scanning optical microscopy (Academic Press, London ; Orlando, 1984).

[4] C.L. Zheng et al, in preparation

[5] CESCOR and CETCOR by CEOS GmbH.

[6] Funding is acknowledged from the Australian Research Council Grants DP110104734 and LE0454166.

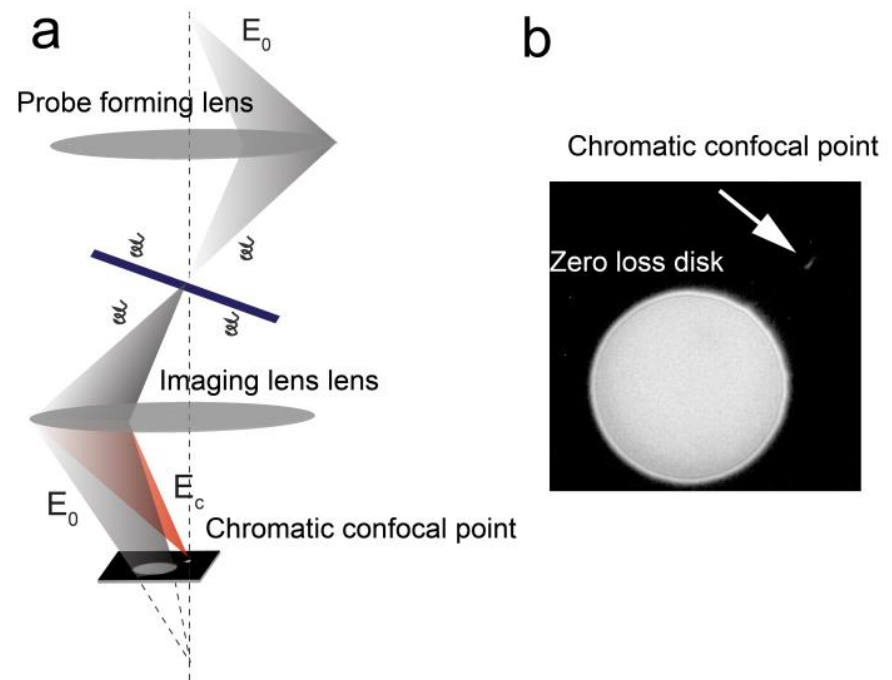

Figure 1. (a) Schematic showing the Off-axis Chromatic SCEM mode. (b) The intensity distribution in the image plane after scattering from amorphous carbon in the off-axis chromatic SCEM mode.
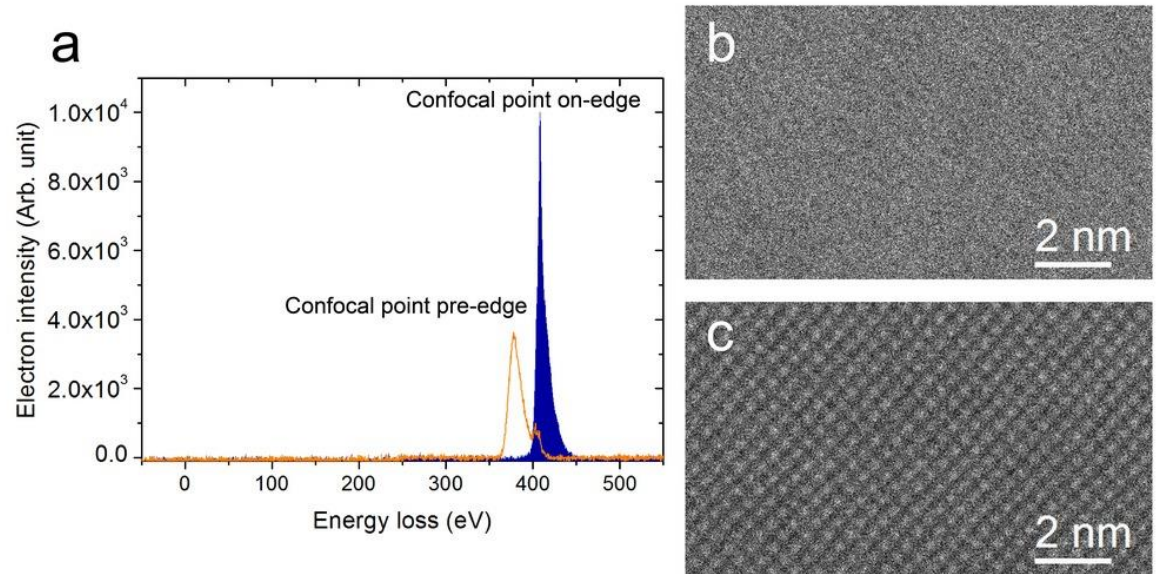

Figure 2. (a) EEL spectra (measured using a post-column spectrometer) arising from electrons around the confocal point in off-axis SCEM. Two separate spectra corresponding to two different focal conditions are shown overlaid - one focus corresponds to the $\mathrm{Sc}_{2,3}$ pre-edge and the other to the $\mathrm{Sc} \mathrm{L}_{2,3}$ on-edge. (b) (c) Off-axis SCEM image using (b) pre-edge and (c) on-edge core loss electrons of Sc $\mathrm{L}_{2,3}$ in $\mathrm{DyScO}_{3}$ (no post-column spectrometer is used). 